\title{
Tracking studies of the Compact Linear Collider collimation system
}

\author{
I. Agapov, H. Burkhardt, and D. Schulte \\ CERN, CH-1211 Geneva 23, Switzerland \\ A. Latina \\ Fermi National Laboratory, Batavia, Illinois 60510, USA \\ G. A. Blair and S. Malton \\ John Adams Institute at Royal Holloway University, London, Egham, Surrey TW20 OEX, United Kingdom \\ J. Resta-López \\ John Adams Institute at Oxford, Oxford OX1 3RH, United Kingdom
}

(Received 12 May 2009; published 10 August 2009)

\begin{abstract}
A collimation system performance study includes several types of computations performed by different codes. Optics calculations are performed with codes such as MADX, tracking studies including additional effects such as wakefields, halo and tail generation, and dynamical machine alignment are done with codes such as PLACET, and energy deposition can be studied with BDSIM. More detailed studies of hadron production in the beam halo interaction with collimators are better performed with GEANT4 and FLUKA. A procedure has been developed that allows one to perform a single tracking study using several codes simultaneously. In this paper we study the performance of the Compact Linear Collider collimation system using such a procedure.
\end{abstract}

DOI: 10.1103/PhysRevSTAB.12.081001

PACS numbers: 29.27.Bd

\section{INTRODUCTION}

Collimation systems are an essential part of high-energy colliders. Beam collimation systems must reduce the background in the detectors, removing the beam halo, and ensure machine protection by minimizing the activation and damage of sensitive accelerator components. A careful design of a collimation system must take into account not only the particles traversing the collimators but also additional effects such as secondary particle production, deflecting kicks induced by wakefields in the collimators, and response to element misalignments.

We begin by discussing the design of the Compact Linear Collider (CLIC) collimation system using the optics code MADX [1]. Further tracking studies including additional effects such as wakefields and dynamical machine alignment are performed with codes such as PLACET [2]. PLACET also contains the HTGEN [3] module which allows for halo and tail generation by beam-gas scattering in the tracking. The code BDSIM [4] has been developed to study energy deposition in beam lines, such as the beam delivery system (BDS) of linear colliders. BDSIM extends the abilities of GEANT4 [5] to allow for fast geometry building and fast tracking in accelerator components. More detailed studies of the beam line interactions may also be made using stand-alone models in GEANT4 (which could also be included within BDSIM) or FLUKA [6].

We introduce PLACET for its capability to calculate wakefield kicks in collimators, and describe a study that applies the wakefield calculations of PLACET to energy deposition studies using BDSIM.

The tracking in BDSIM and PLACET has been carefully benchmarked against other high accuracy tracking codes $[7,8]$, with agreement between the CLIC spot sizes obtained at the interaction point (IP) at the sub-nm level over several $\mathrm{km}$ of tracking.

\section{COLLIMATION SYSTEM DESIGN WITH MADX}

The first postlinac collimation section of the CLIC BDS is dedicated to energy collimation. The energy collimation depth is determined by failure modes in the linac [9]. A spoiler/absorber scheme, located in a region with nonzero horizontal dispersion, is used for intercepting mis-steered or errant beams with energy deviation larger than about $1.3 \%$ of the nominal energy.

Downstream of the energy collimation section, a dispersion-free section, containing eight spoilers and eight absorbers, is dedicated to the cleaning of the transverse halo of the beam, thereby reducing the experimental background at the IP. Figure 1 shows the square root $\beta$ and horizontal dispersion functions in the various sections of the BDS.

For efficient collimation in the transverse phase spaces $x-x^{\prime}$ and $y-y^{\prime}$, the phase advance between collimators and with respect to the IP is important. The transverse phase advance between the spoiler positions and the IP is generally set to be $n \pi$ or $(1 / 2+n) \pi$, with $n=0,1,2,3, \ldots$. The IP is at $\pi / 2$ or $(1 / 2+n) \pi$ phase advance from the 


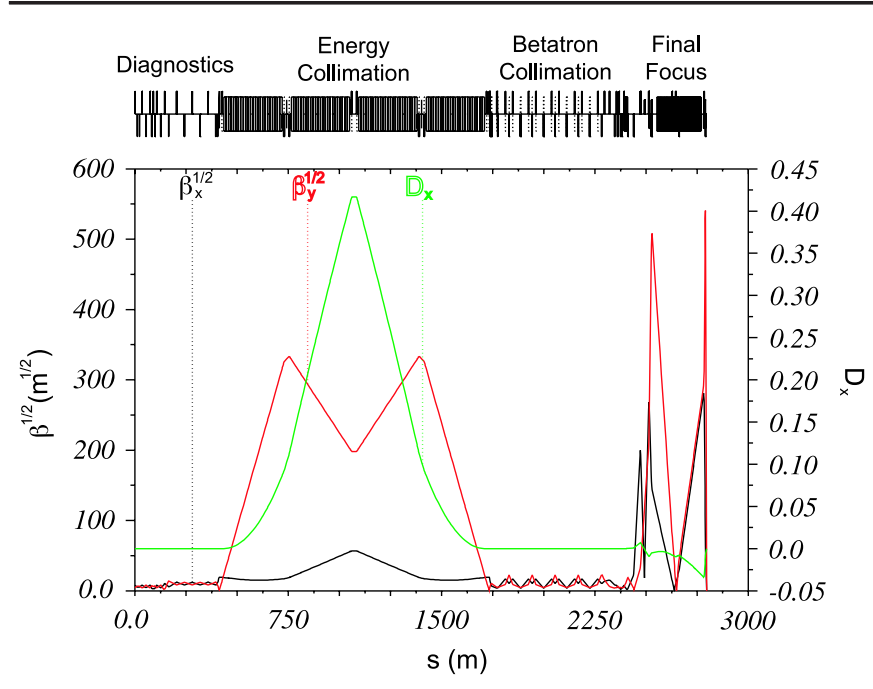

FIG. 1. (Color) Horizontal dispersion and square root of the betatron functions for the CLIC BDS.

final doublet (FD). For the latest version of the CLIC BDS lattice, Fig. 2 illustrates the phase advance between the betatron spoilers, spoiler-IP, and spoiler-FD phase advances. The spoilers XSP1 (YSP1) and XSP3 (YSP3) would collimate amplitudes at the FD phase, while the spoilers XSP2 (YSP2) and XSP4 (YSP4) collimate amplitudes at the IP phase.

For linear colliders, the necessary betatron collimation depths are typically determined from the condition that beam particles and synchrotron radiation photons emitted in the final quadrupoles should not hit any magnet apertures on the incoming side of the IP. Ray-tracing calcula-
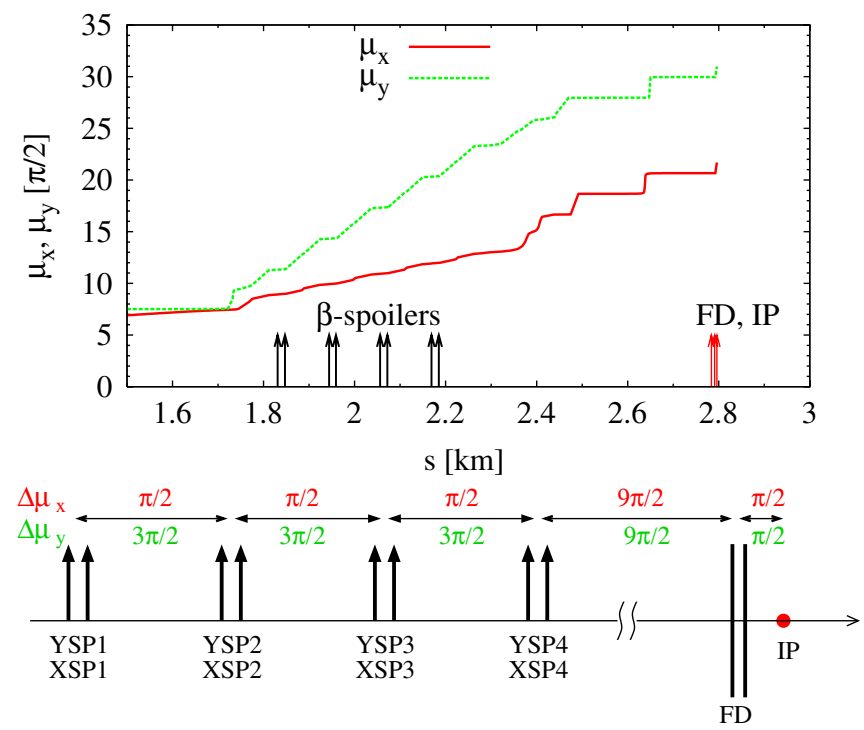

FIG. 2. (Color) Top: horizontal (solid red line) and vertical (dashed green line) phase advance in the betatron collimation section and final focus system of CLIC. Bottom: schematic showing the approximate values of the phase advance between the CLIC betatron spoilers, FD and IP.
TABLE I. CLIC postlinac optics and collimator parameters. Horizontal and vertical $\beta$ functions, horizontal dispersion, horizontal and vertical half gaps, and material. The horizontal and vertical emittances of the beam, $\gamma \epsilon_{x, y}$, are $660 \mathrm{~nm} \mathrm{rad}$, and $20 \mathrm{~nm}$ rad, respectively.

\begin{tabular}{|c|c|c|c|c|c|c|}
\hline Collimator & $\begin{array}{c}\beta_{x} \\
{[\mathrm{~m}]}\end{array}$ & $\begin{array}{c}\beta_{y} \\
{[\mathrm{~m}]}\end{array}$ & $\begin{array}{c}D_{x} \\
{[\mathrm{~m}]}\end{array}$ & $\begin{array}{c}a_{x} \\
{[\mathrm{~mm}]}\end{array}$ & $\begin{array}{c}a_{y} \\
{[\mathrm{~mm}]}\end{array}$ & Material \\
\hline E-SP & 1406.33 & 70681.9 & 0.27 & 3.51 & 25.4 & $\mathrm{Be}$ \\
\hline E-AB & 3213.03 & 39271.5 & 0.416 & 5.41 & 25.4 & $\mathrm{Ti} / \mathrm{Cu}$ \\
\hline$\beta_{y}-\mathrm{SP}$ & 114.054 & 483.253 & 0 & 10.0 & 0.08 & $\mathrm{Be}$ \\
\hline$\beta_{y}-\mathrm{AB}$ & 114.054 & 483.184 & 0 & 1.0 & 1.0 & $\mathrm{Ti} / \mathrm{Cu}$ \\
\hline$\beta_{x}-\mathrm{SP}$ & 270.003 & 101.347 & 0 & 0.08 & 10.0 & $\mathrm{Be}$ \\
\hline$\beta_{x}-\mathrm{AB}$ & 270.102 & 80.9043 & 0 & 1.0 & 1.0 & $\mathrm{Ti} / \mathrm{Cu}$ \\
\hline
\end{tabular}

tions based on this criterion give a collimation depth of $16 \sigma_{x}$ (horizontal) and $70 \sigma_{y}$ (vertical) [10]. For CLIC the protection of the permanent final quadrupole, so-called QD0, against incident particles has been adopted as a safer criterion, i.e., the bore aperture of QD0 determines the actual betatron collimation depth: $10 \sigma_{x}$ (horizontal) and $44 \sigma_{y}$ (vertical) [11]. Table I summarizes the CLIC postlinac collimator parameters. The horizontal aperture for the momentum collimator is set to $a_{x}=D_{x} \delta_{\text {aper }}$, with the energy offset $\delta_{\text {aper }}= \pm 1.3 \%$.

\section{COLLIMATOR WAKEFIELDS}

Wakefields in the BDS can cause severe single or multibunch effects leading to luminosity loss. Jitter amplification and emittance growth can be driven by wakefields and degrade the electron or positron beam quality at the IP with a consequent luminosity drop.

The main contributions to wakefields in the BDS are: (i) geometric and resistive wall wakefields of the tapered and flat parts of the collimators (pipe radius changes and small apertures); (ii) resistive wall wakes of the beam pipe, which are especially important in the regions of the final quadrupoles (where the $\beta$ functions are very large); (iii) electromagnetic modes induced in crab cavities. Crab cavities are needed to rotate the bunches in order to compensate for a crossing angle at the IP. In this paper we concentrate on single bunch effects of the collimator transverse wakefields.

\section{TRACKING AND ALIGNMENT STUDIES WITH PLACET}

PLACET is a tracking code that simulates the dynamics of a particle beam in future high-energy linear colliders. It can simulate bunch compressors, the main linac, and beam delivery systems as well as structures specific to CLIC, such as the drive beam. It takes into account realistic effects such as long- and short-range wakefields in the accelerating structures, geometric and resistive wall wakefields in the collimators, and incoherent synchrotron radia- 
TABLE II. Luminosity variation for different collimator gaps. Beam sizes were obtained from a Gaussian fit to the particle distribution at the IP. The collimator gap is set for both horizontal and vertical collimators simultaneously. $L_{0}$ is the calculated luminosity without wakefields.

\begin{tabular}{lccccc}
\hline \hline Gap $(\mu \mathrm{m})$ & 60 & 70 & 80 & 90 & 100 \\
$L / L_{0}$ & 0.9713 & 0.9486 & 1.0165 & 1.0097 & 1.0314 \\
\hline \hline
\end{tabular}

tion emission in all magnets and coherent synchrotron radiation emission in the bends. In bends, when the wavelength of the emitted radiation is comparable with the beam dimensions, electron bunches can radiate coherently. The coherent radiation force might drive an instability and induce a significant growth of the bunch emittance and needs to be treated specifically. PLACET allows the simulation of static and dynamic misalignments, ground motion, and other machine imperfections. To control the simulation, PLACET provides a programmable interface based on TCL/TK [12] and OCTAVE [13]. A number of correction schemes make it possible to test and reduce the emittance growth to be expected for given prealignment errors.

\section{Collimator wakefields in PLACET}

A module for the calculation of the collimator wakefields in different regimes has been implemented in the PLACET tracking code. Given a set of parameters such as material conductivity, tapering length, and angle, the code first determines the type of regime (geometric: inductive, diffractive or intermediate, and resistive: long- or shortrange, dc or ac conductivity), then evaluates the kick on a bunch particle as a function of its longitudinal (and transverse, if the collimator is flat and there is a quadrupole component of the wake) position and applies it to the particle accordingly. The geometric component of the wake has the same shape as the bunch and is usually negligible with respect to the overall contribution given by the resistive wall. A more detailed description is presented in [14].
The luminosity of the beam is proportional to $1 / \sqrt{\sigma_{x}^{*} \sigma_{y}^{*}}$, where $\sigma^{*}$ is the beam spot size at the IP. Tracking the core beam in PLACET for different collimator gap sizes, we can see the effect of wakefields on the luminosity. Table II shows the relative luminosity for a range of collimator gaps, calculated solely from the change in beam size at the IP. We see that there is a general trend of increasing luminosity as the gap is increased. In particular, we note that for collimator gaps of $80 \mu \mathrm{m}$ and above, the luminosity inclusive of wakefields appears to be greater than the luminosity without wakefields $\left(L / L_{0}>1\right)$. Whether this is a real effect or an artifact of the simulation is something that requires further investigation. Also effects such as beam jitter-which tends to decrease the luminosity in the presence of wakefields [14] — and the "pinch" effect at the interaction point need to be included in order to determine the exact effect on the luminosity. A more accurate calculation may be obtained using a code such as GUINEAPIG [15].

\section{HALO AND TAIL GENERATION WITH HTGEN}

Halo particles in linear colliders can result in significant losses and serious background which may reduce the overall performance. Even if most of the halo is stopped by collimators, the secondary muon background may still be significant. It is therefore important to include in collimation studies halo generation and tracking. The halo generation is done with the HTGEN code which is fully interfaced and integrated in PLACET, which allows the tracking of the halo together with the beam core. We present here a short description of the halo and tail generation with focus on what is relevant for the beam delivery system. More information can found in [3]. Halo particles can be produced anywhere in the system. Here we assume that beams are cleaned before they are accelerated in the CLIC linac so that we only need to consider the extra halo production in the linac and the BDS. HTGEN generates halo by beam-gas scattering. On the level of simulations with

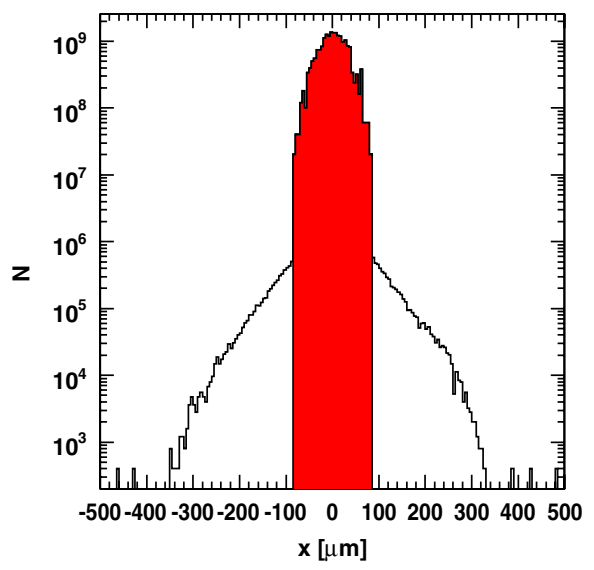

FIG. 3. (Color) Transverse beam profiles at the CLIC BDS entrance. The core beam is shown in red. 
PLACET-HTGEN, it is sufficient to specify the rest gas pressure and composition and to enable background tracking. It is possible to specify gas parameters for each element or for groups of elements using for-loop constructs in TCL/TK as input to PLACET.

Figure 3 shows typical transverse distributions obtained from HTGEN+PLACET at the entrance to the BDS [3]. The flux of halo particles which will impact on the collimators will depend on the collimator settings and details of the lattice parameters including imperfections and misalignment. Based on preliminary collimation studies and simulations under rather idealistic assumptions, we find for 10 nTorr CO both in the CLIC linac and BDS, that a fraction of about $2 \times 10^{-4}$ of all particles will have large amplitudes and hit the spoilers in the BDS section. With $1.24 \times 10^{12}$ particles per train, this would translate into a flux of $2.4 \times 10^{8}$ particles per train impacting on the spoilers. At $1.5 \mathrm{TeV}$, we expect that a fraction of about $9 \times 10^{-4}$ of these particles produce secondary muons, resulting in a flux of about $2 \times 10^{5}$ muons per train, many of which would be seen as background in the detector in the interaction region. Reducing the muon flux would require very massive shielding, of the order of $100 \mathrm{~m}$ of (magnetized) tunnel fillers, to be effective [16].

\section{ENERGY DEPOSITION AND SECONDARY PRODUCTION STUDIES WITH BDSIM}

Typical loss maps consider a particle lost if it interacts with the beam line aperture in any way. A program such as GEANT4 can then be used to examine areas of interest in more detail. BDSIM combines particle tracking and secondary particle production to generate detailed loss maps for whole beam line more efficiently.

We track a beam halo through the CLIC BDS. The halo is divided into concentric ellipses in $x-x^{\prime}$ and $y-y^{\prime}$ phase space independently, where each ellipse is of thickness $5 \sigma_{x^{(l)}}$ or $10 \sigma_{y^{(1)}}$. These ellipses then cover the whole phase space from $0-40 \sigma_{x^{(1)}}$ and $0-190 \sigma_{y^{(1)}}$. The energy and longitudinal profiles are chosen to be the same as for the core beam: a flat distribution of width $1 \%$ about the nominal beam energy of $1496 \mathrm{GeV}$, and a Gaussian of width $44 \mu \mathrm{m}$, respectively. The particle distribution within each ellipse is uniform, and each ellipse contains 10000 particles; this approximates a $1 / r$ density profile in each phase space, and gives a total halo population of 1520000 . From Sec. V we have a halo population of $2 \times 10^{-4}$ of the bunch charge, or $8 \times 10^{5}$ particles. This is approximately half of the amount simulated. Alternatively, if we assume that CLIC will achieve a similar level of halo to that which the Stanford Linear Collider managed in its later runsabout $0.1 \%$ of the bunch charge - then, for a bunch of $4 \times$ $10^{9}$ particles [17], we are simulating approximately $40 \%$ of the halo population. The numbers which follow have not been scaled to account for this.

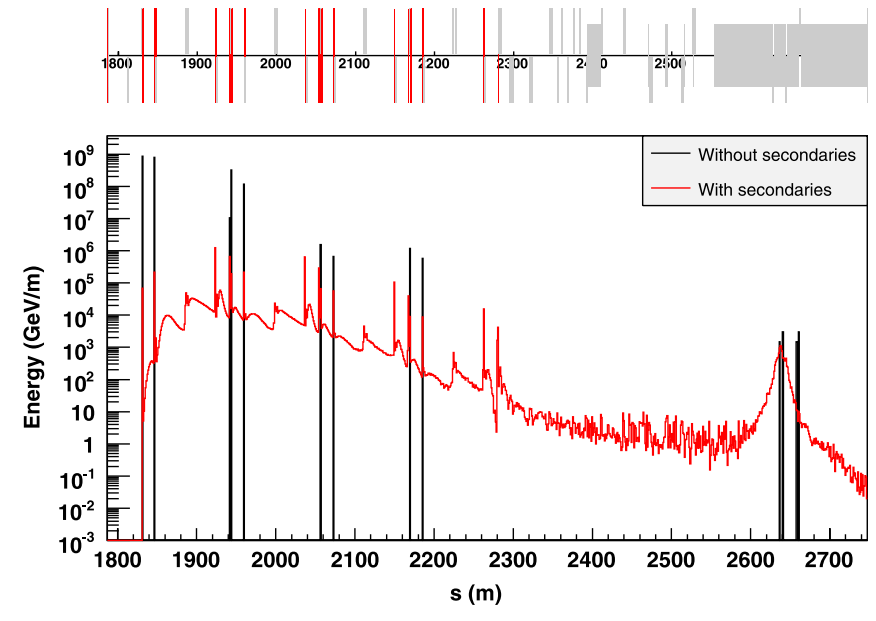

FIG. 4. (Color) Energy deposition along the beam line from halo particles, with (red) and without (black) secondary particle production and scattering. Losses from synchrotron radiation have not been included.

Figure 4 shows the energy deposition profile of the beam halo in the CLIC BDS. The black histogram is produced assuming that particles that hit any element of the beam line are completely absorbed at that point, while the red histogram includes multiple scattering and secondary particle production. In this instance, it is seen that the peak load on the beam spoilers is reduced by up to 4 orders of magnitude in the case of YSP1 (the first betatron spoiler in the line). We note that there are no direct impacts on the thick absorbers; losses occur on the absorbers only when secondary particles are included. There are a small number of primary halo particles lost in the final focus system; to correct this will require either a tightening of the collimator gaps or a redesign of the lattice optics. It should be noted that the collimator geometry employed in this study using BDSIM does not include tapering: the aperture is set to the minimum gap for the length of the collimator, therefore it is possible that the collimator efficiency is somewhat optimistic in this study.

\section{PLACET-BDSIM INTEGRATION}

Halo particles that are close to the walls of the beam pipe may be kicked by the collimator wakefields and interact with the beam-pipe material, producing secondary particles. A single simulation code that implements wakefields, tracking, and secondary particle generation does not exist. On the one hand, a code like BDSIM is designed to track single particles and their secondaries deriving from the interactions with the materials, but does not include intrabunch interactions; on the other hand, a code such as PLACET takes into account collective effects but does not simulate the interactions of the particles with the walls of the beam line. Combining the abilities of BDSIM and PLACET enables an accurate simulation of the generation of secondary particles and their tracking in components 


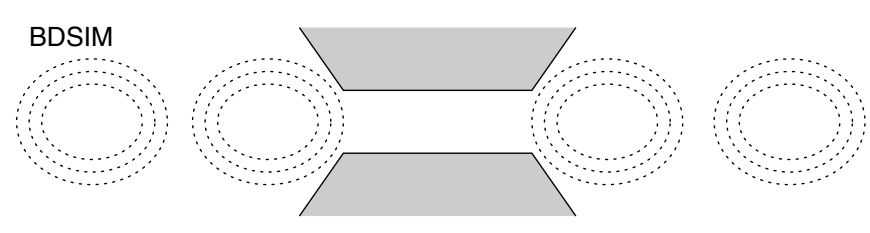

PLACET

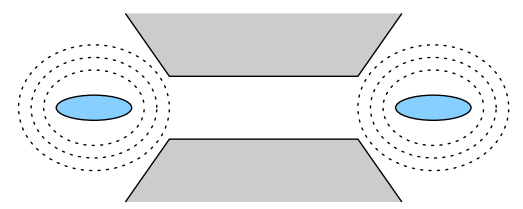

(2)

(3)

(4)

FIG. 5. (Color) Schematics of the PLACET-BDSIM interface. The tracking proceeds through four steps: (1) BDSIM tracks the halo particles (shown dotted) while PLACET tracks the bunch core (shown in blue), along the BDS; (2) at the first collimator entrance, halo particles are sent from BDSIM to PLACET; (3) PLACET tracks core and halo through the collimator, taking into account wakefield effects; then, it sends back the wake kicks to BDSIM. BDSIM waits until it receives the wake kicks from PLACET; then, it tracks the halo particles through the collimators, considering scattering and secondary particle generation, and finally applies the wake kicks to the halo particles; (4) the tracking continues until the next collimator.

such as the collimators, taking into account the most relevant collective effects. We developed a method to achieve this. It consists of interfacing the PLACET tracking code and BDSIM, based on the following idea: the tracking is performed in parallel by both codes (see Fig. 5). BDSIM tracks halo particles, while PLACET tracks core particles. When BDSIM reaches an element where it is desired to include the effects of wakefields, BDSIM sends to PLACET the halo particles to perform the calculations of the wakefield kick; then PLACET reports the resulting kick angles for each particle back to BDSIM, which applies the kicks and continues the tracking.

\section{A. Combined tracking}

Performing this parallel tracking without secondary particle production switched on in BDSIM, we can determine the effect of wakefields on particle losses from the beam halo. Halo losses with and without wakefield effects are shown in Fig. 6. We see that the wakefields lead to approximately double the amount of losses on the last two vertical spoilers; there is no increase in losses on the horizontal spoilers. Losses of primary halo particles in the final focus system remain. Secondary particle losses in this region are dominated by photons and positrons which are lost in the bending magnets.

Figure 7 shows the same energy deposition profiles with secondary particle production switched on. Losses on the collimators do not differ significantly between the two cases; however, secondary losses occur closer to the spoiler when wakefields are included. This may have implications for quadrupole magnet protection in these areas.

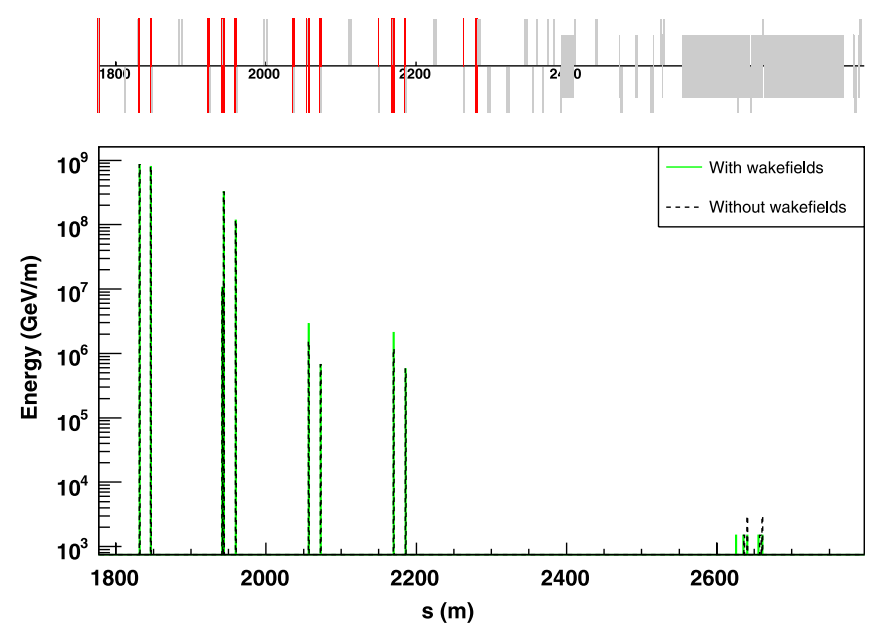

FIG. 6. (Color) Energy deposition along the beam line from halo particles, with (green) and without (black) wakefield interactions. All particles hitting a collimator are absorbed totally at that point.

The core beam geometry is roughly flat, with an aspect ratio of approximately 10:1. This causes the wakefields to be much larger in the vertical direction than in the horizontal. Figures 8 and 9 show the distribution of particles by which ring they occupy in horizontal and vertical phase space, respectively. The initial distribution described in Sec. VI consists of $10^{4}$ particles per ring. The distributions in these figures are the projections of the $2 \mathrm{D}$ distributionthis would lead to there being $1.9 \times 10^{5}$ particles per $5 \sigma$ bin in Fig. 8 and $9 \times 10^{4}$ particles per $10 \sigma$ bin in Fig. 9 . The values at low- $x(y)$ are lower than this due to loses in the high- $y(x)$ region, and because of the different binning.

The distribution in horizontal phase space does not vary significantly with either wakefield or secondary particle
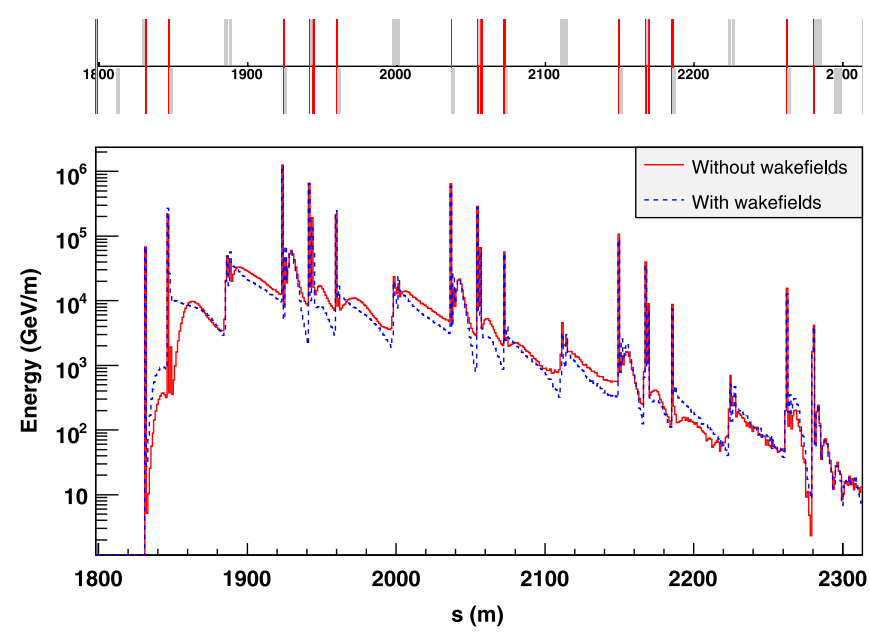

FIG. 7. (Color) Energy deposition along the beam line from halo particles, with (blue) and without (red) wakefield interactions. In this instance, secondary particle production and scattering has been switched on. 


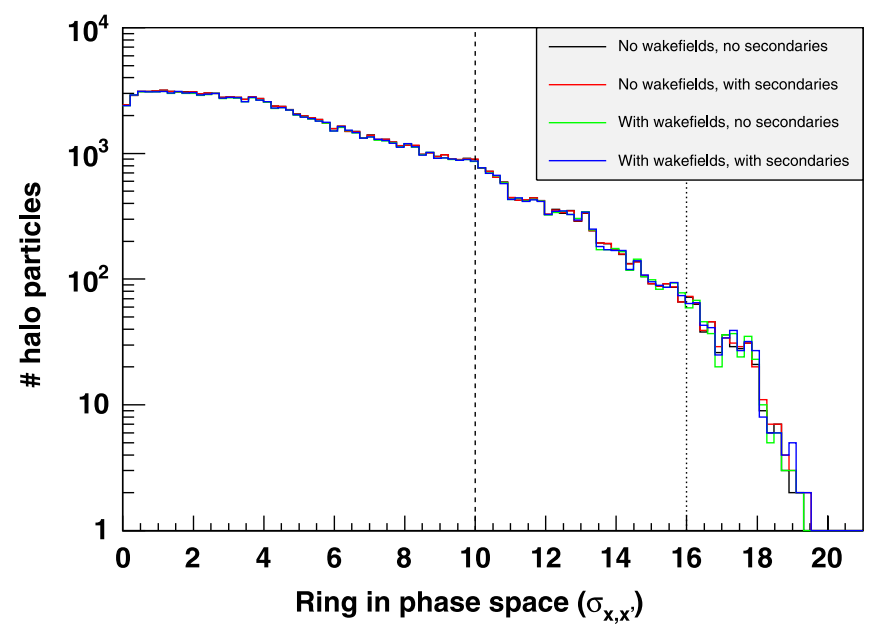

FIG. 8. (Color) Amplitude of particles in horizontal phase space at the entrance to QD0. The vertical dotted lines are located at the collimation depth limits of 10 and $16 \sigma_{x^{(1)}}$.

production for the tighter $10 \sigma$ limit. However, for the looser $16 \sigma$ limit the number of particles at an unacceptably high $\sigma$ value doubles when secondary particles are turned on. In all cases we have not counted particles which are well outside the bore of the final magnet, as these will not contribute to backgrounds in the detector.

The distributions in vertical phase space show some variation with each process. Turning on wakefields decreases the number of particles above the collimation depth, while turning on secondary particles increases this number. The number of particles above the collimation depth criteria is quantified in Table III. We have not corrected for double counting here, where a particle may violate the collimation depth limit in both $x$ and $y$, and so the total number of particles outside the limit will be less than the sum of the horizontal and vertical values.

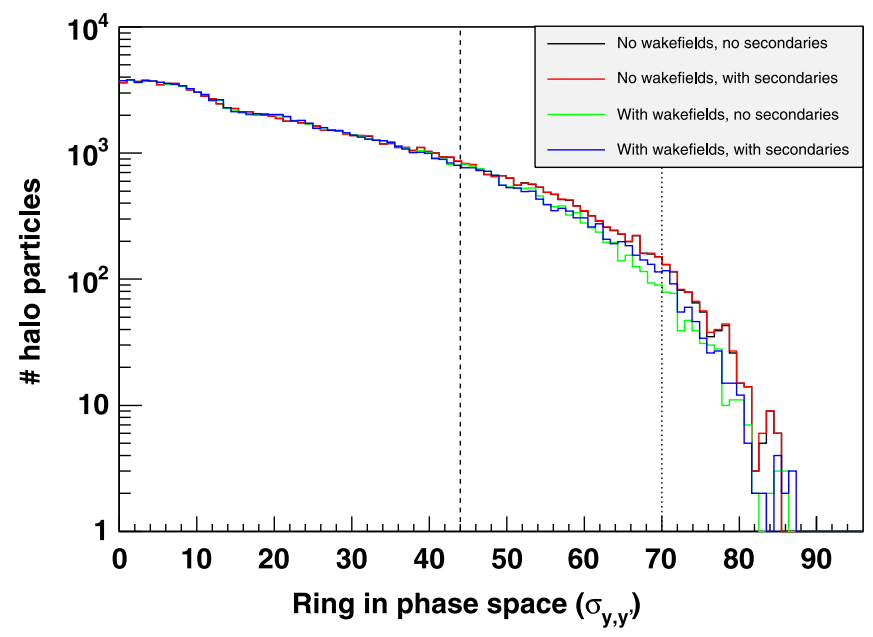

FIG. 9. (Color) Amplitude of particles in vertical phase space at the entrance to QD0. The vertical dotted lines are located at the collimation depth limits of 44 and $70 \sigma_{y^{(/)}}$.
TABLE III. The number of particles at the entrance to QD0 outside the collimation depth. The collimator gap (both horizontal and vertical) is set to $80 \mu \mathrm{m}$. The initial particle distribution is described in Sec. VI. "Wf" indicates wakefields, "Sec." indicates secondary particle production.

\begin{tabular}{cccccc}
\hline \hline Wf & Sec. & $>10 \sigma_{x^{(l)}}$ & $>16 \sigma_{x^{(\prime)}}$ & $>44 \sigma_{y^{(\prime)}}$ & $>70 \sigma_{y^{(l)}}$ \\
\hline Off & Off & 9943 & 419 & 14508 & 873 \\
Off & On & 11663 & 733 & 16000 & 1068 \\
On & Off & 9847 & 414 & 11753 & 471 \\
On & On & 11411 & 890 & 12529 & 729 \\
\hline \hline
\end{tabular}

\section{B. Collimator gaps}

We have shown that the inclusion of these extra processes leads to an overall decrease in the number of particles outside the collimation depth. It may therefore be desirable to increase the collimator gaps-if this can be achieved without unduly affecting the expected backgrounds in the detector region or the impact on QD0 - as we have shown that this will improve luminosity by reducing the wakefields.

As would be expected, a narrower collimator gap leads to higher losses on the early spoilers by virtue of the geometry. Conversely, losses between the collimators tend to increase later in the beam line as the collimator gap is opened, as shown in Fig. 10. However, it is unclear how much of the change in energy deposition comes from the change in the physical aperture and how much is due to the change in wakefields from the altered geometry.

No significant difference occurs between the end of the collimation section and the beginning of the final dispersion-correction region. Within the bending magnets the losses increase with collimator gap, as shown in Fig. 11.
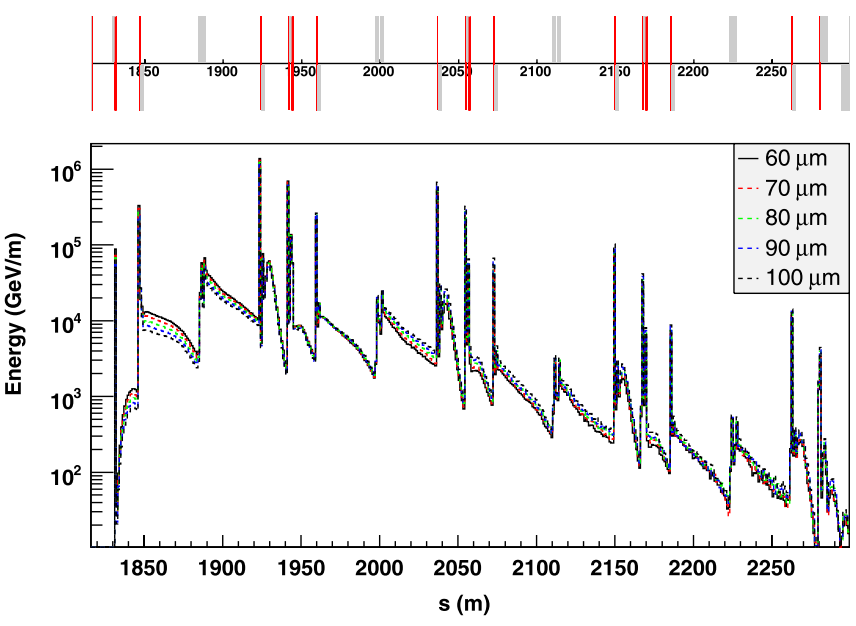

FIG. 10. (Color) Energy deposition along the beam line from halo particles for different collimator gap settings. Both wakefields and secondary particle production are switched on. A zoomed view of the spoiler/absorber region. 

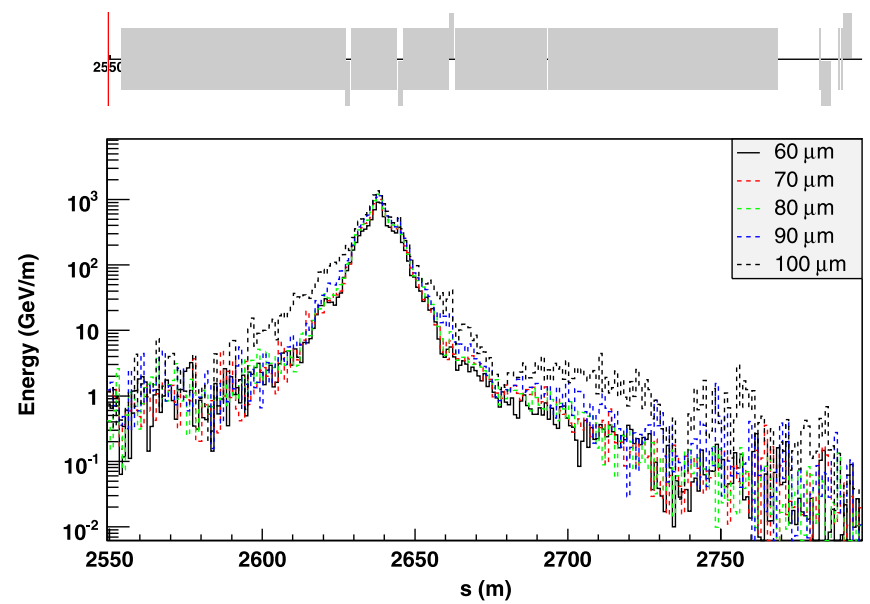

FIG. 11. (Color) Energy deposition along the beam line from halo particles for different collimator gap settings. Both wakefields and secondary particle production are switched on. A zoomed view of the interaction region. The IP is located at $2796 \mathrm{~m}$.

The total number of particles arriving at the entrance to the final focusing magnet QD0 for each collimator gap with and without wakefields and secondary particles is given in Table IV. We observe that the switching on and off of the new processes varies the number of particles arriving at the magnet by approximately 1000 particles. Opening the collimator gap increases the number of particles by 20000-30000 for each $10 \mu \mathrm{m}$.

Taking the case with both wakefields and secondary particles, we can then perform this analysis for the various collimator gaps as we did earlier for the energy deposition. The particle distributions in vertical phase space are shown in Fig. 12, and the number of particles for each gap is given in Table V. Table $\mathrm{V}$ also includes the numbers without wakefields for comparison. The number of particles outside the collimation depth increases with the widening of the collimator gap, as would be expected. We note that the decrease in errant particles caused by the wakefields is not large enough to compensate for this, at least for the stepsize granularity which we have chosen.

TABLE IV. The total number of particles at the entrance to QD0 for various collimator gap settings with and without wakefield interactions ("Wf") and secondary particle production ("Sec.").

\begin{tabular}{lcccc}
\hline \hline Gap $(\mu \mathrm{m})$ & $\begin{array}{c}\text { No Wf } \\
\text { No Sec. }\end{array}$ & $\begin{array}{c}\text { No Wf } \\
\text { Sec. }\end{array}$ & $\begin{array}{c}\text { Wf } \\
\text { No Sec. }\end{array}$ & $\begin{array}{c}\text { Wf } \\
\text { Sec. }\end{array}$ \\
\hline 60 & 59773 & 59856 & 57116 & 57188 \\
70 & 82550 & 82597 & 80373 & 80865 \\
80 & 107807 & 107912 & 106019 & 106547 \\
90 & 136428 & 136549 & 134987 & 135199 \\
100 & 167842 & 168016 & 166806 & 167192 \\
\hline \hline
\end{tabular}

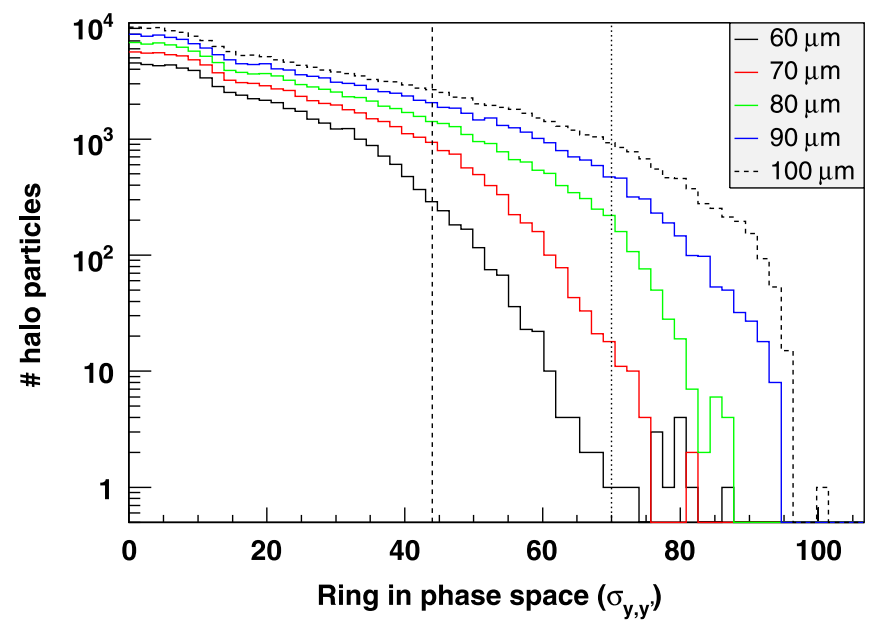

FIG. 12. (Color) Particle amplitudes in vertical phase space at the entrance to QD0 for varying collimator gaps. Wakefields and secondary particle production are both switched on. The vertical dotted lines are located at the collimation depth limits of 44 and $70 \sigma_{y^{(\prime)}}$.

TABLE V. The number of particles at the entrance to QD0 outside the collimation depth. Secondary particle production and scattering are switched on.

\begin{tabular}{lcccc}
\hline \hline Gap $(\mu \mathrm{m})$ & $\begin{array}{c}>44 \sigma_{y^{(\prime)}} \\
\text { No Wf }\end{array}$ & $\begin{array}{c}>44 \sigma_{y^{(\prime)}} \\
\text { Wf }\end{array}$ & $\begin{array}{c}>70 \sigma_{y^{(\prime)}} \\
\text { No Wf }\end{array}$ & $\begin{array}{c}>70 \sigma_{y^{(\prime)}} \\
\text { Wf }\end{array}$ \\
\hline 60 & 2451 & 1253 & 8 & 13 \\
70 & 6519 & 5307 & 60 & 36 \\
80 & 16000 & 12529 & 1068 & 729 \\
90 & 22116 & 20468 & 3131 & 2249 \\
100 & 34537 & 33512 & 6962 & 7117 \\
\hline \hline
\end{tabular}

\section{CONCLUSION}

Secondary particle generation and multiple scattering reduces locally the amount of energy deposited in collimators due to beam halo impacts by several orders of magnitude. While a standard loss map shows losses to be almost entirely confined to the spoilers-barring a few particles in the final focus which must be excluded by a redesign of the beam line optics-losses due to secondary particles extend all the way to the interaction point. This serves to increase the number of particles arriving at the final focusing magnet, which may have a detrimental effect on detector backgrounds, and on the magnet itself.

The inclusion of wakefield effects on full halo tracking produces a small but potentially significant decrease in the number of particles outside the required collimation depth, although this decrease is partially mitigated by the increase from secondary particles. The reduction in particle numbers is not of sufficient extent that the collimator gaps may be increased significantly.

The requirement that no halo particles impact upon the final focusing quadrupole is not met using this current 
CLIC collimation scheme, regardless of the additional effects included in the simulation. The normal synchrotron-radiation-fan tolerance is better met as would be expected by the looser constraints it requires. Decreasing the collimator gaps can reduce these backgrounds by up to $90 \%$ for the $44 \sigma$ limit, and by $99 \%$ for the $70 \sigma$ limit, although it has been shown that this would have a detrimental effect on the luminosity, due to increased wakefields (see Table II and [18-20]).

Further work to quantify the acceptable limits in either case is required to determine whether these particles will cause issues for CLIC. The techniques reported here will provide important contributions to the optimization of the final CLIC collimation system design.

\section{ACKNOWLEDGMENTS}

This work was supported in part by the STFC LC-ABD Collaboration and by the Commission of European Communities under the 6th Framework Programme Structuring the European Research Area, Contract No. RIDS-011899.

[1] H. Grote and F. Schmidt, in Proceedings of the 20th Particle Accelerator Conference, Portland, Oregon, 2003 (IEEE, Piscataway, NJ, 2003), FPAG014.

[2] A. Latina et al., in Proceedings of the 10th European Particle Accelerator Conference, Edinburgh, Scotland, 2006 (EPS-AG, Edinburgh, Scotland, 2006), WEPCH140.

[3] H. Burkhardt et al., EUROTeV-Report-2008-076, 2008.

[4] I. Agapov, G. A. Blair, S. Malton, and L. Deacon, Nucl. Instrum. Methods Phys. Res., Sect. A 606, 708 (2009).

[5] S. Agostinelli et al., Nucl. Instrum. Methods Phys. Res., Sect. A 506, 250 (2003).

[6] A. Fassò et al., in Proceedings of the 3rd Workshop on Simulating Accelerator Radiation Environments (SARE 3), edited by H. Hirayama, KEK Proceedings No. 97-5 (KEK, Tsukuba, Japan, 1997), p. 32.

[7] S. Redaelli et al., in Proceedings of the 8th European Particle Accelerator Conference, Paris, 2002 (EPS-IGA and CERN, Geneva, 2002), MOPRI050.

[8] D. Schulte, P. G. Tenenbaum, N. Walker, A. Wolski, and M. Woodley, http://documents.cern.ch/cgi-bin/setlink? base $=$ preprint $\&$ categ $=$ cern $\&$ id $=$ cern-open-2002-059 (2002).

[9] D. Schulte and F. Zimmermann, in Proceedings of the 19th Particle Accelerator Conference, Chicago, Illinois, 2001 (IEEE, Piscataway, NJ, 2001); CLIC Note 492, 2001; CERN Report No. CERN-SL-2001-034 (AP) (2001).

[10] F. Jackson, CLIC Workshop, 2008, http://indico.cern.ch/ conferenceDisplay.py? confId $=30383$.

[11] R. Tomás (private communication).

[12] J. K. Ousterhout, An Introduction to TCL and TK (AddisonWesley, Reading, MA, 1993).

[13] J.W. Eaton, GNU OCTAVE Manual (Network Theory Limited, Bristol, U.K., 2002), ISBN 0-9541617-2-6.

[14] G. Rumolo, A. Latina, and D. Schulte, in Proceedings of the 10th European Particle Accelerator Conference, Edinburgh, Scotland, 2006 (EPS-AG, Edinburgh, Scotland, 2006), MOPLS096.

[15] D. Schulte, Ph.D. thesis, University of Hamburg [TESLA Report No. TESLA-97-08, 1996].

[16] H. Burkhardt, G. A. Blair, and H.J. Schreiber, in Proceedings of the 8th European Particle Accelerator Conference, Paris, 2002 (EPS-IGA and CERN, Geneva, 2002), OPRI051.

[17] F. Tecker et al., CLIC-Note-764, 2008.

[18] R. Assmann, in Proceedings of the Particle Accelerator Conference, Vancouver, BC, Canada, 1997 (IEEE, New York, 1997).

[19] G. Rumolo, A. Latina, and D. Schulte, EuroTevReport-2006-026, 2006.

[20] J. L. Fernandez-Hernando, S. Molloy, J. D. A. Smith, and N.K. Watson, in Proceedings of the 11th European Particle Accelerator Conference, Genoa, 2008 (EPS-AG, Genoa, Italy, 2008), WEPP163. 